\title{
The Effects of Muscle Relaxation Therapy in Reducing Head and Neck Cancer Postoperative Induced Fatigue and Sleep Quality: A Randomized Controlled Trial
}

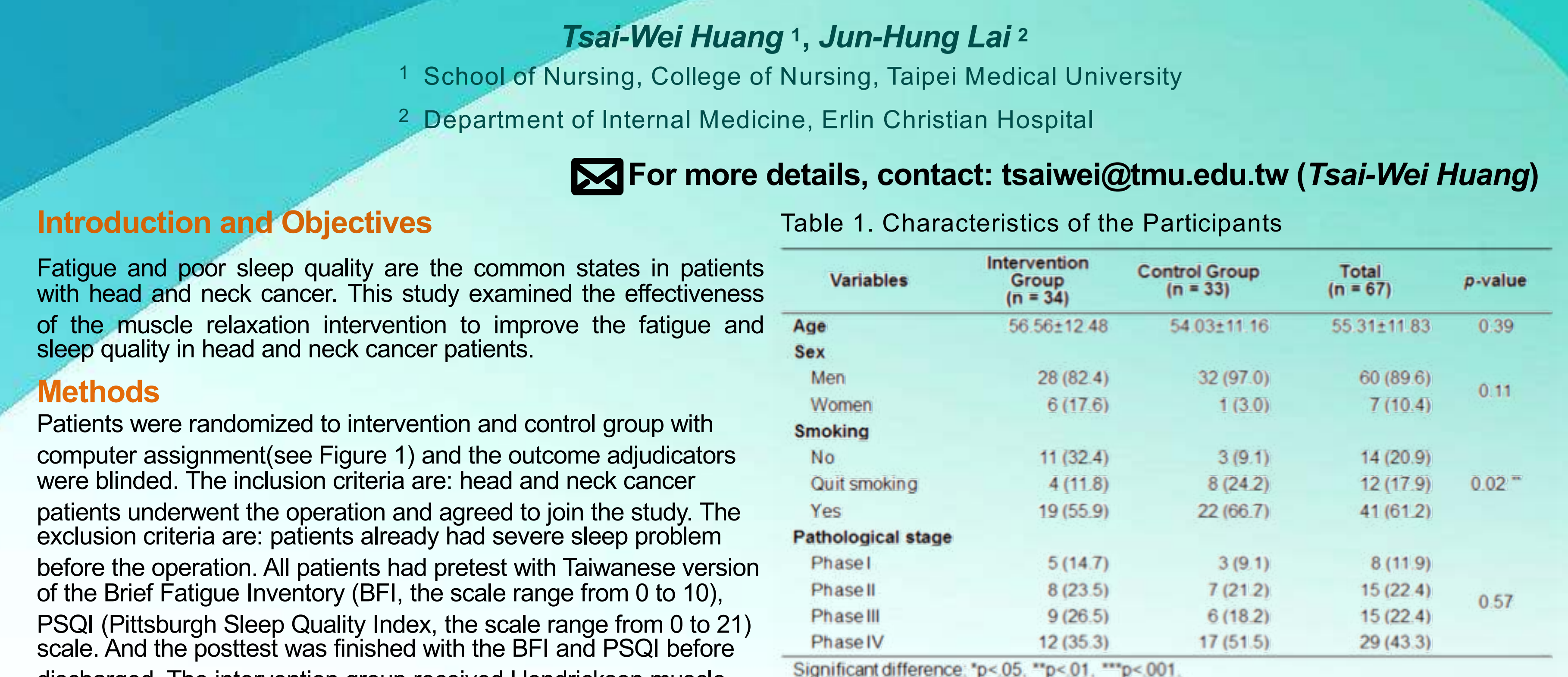

discharged. The intervention group received Hendrickson muscle relaxation therapy for 15 minutes every night from the Day 3 of transfer from intensive care unit until they were discharged from hospital and the control group received regular care. In addition of fatigue and sleep quality, we also observed and recorded the all patients' depression status on each day (Visual Analogue Scale, the range from 0 to 10$)$.

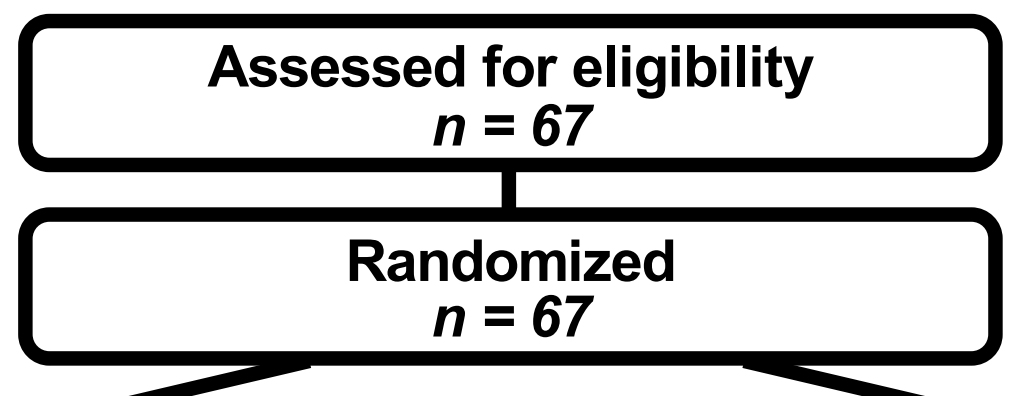

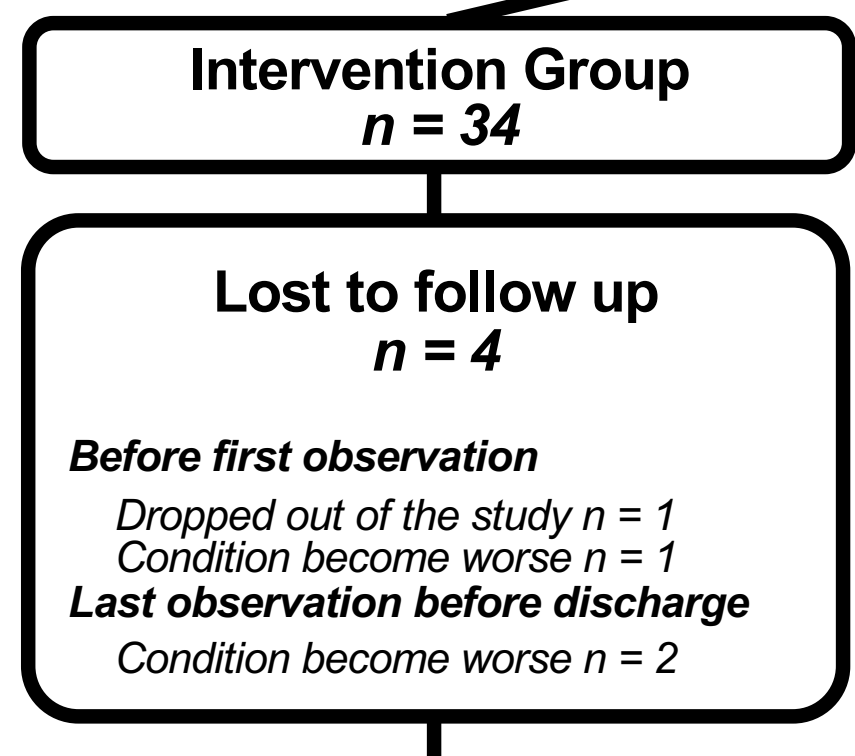

Analysed $\mathbf{n}=30$

Excluded from analysis $n=0$

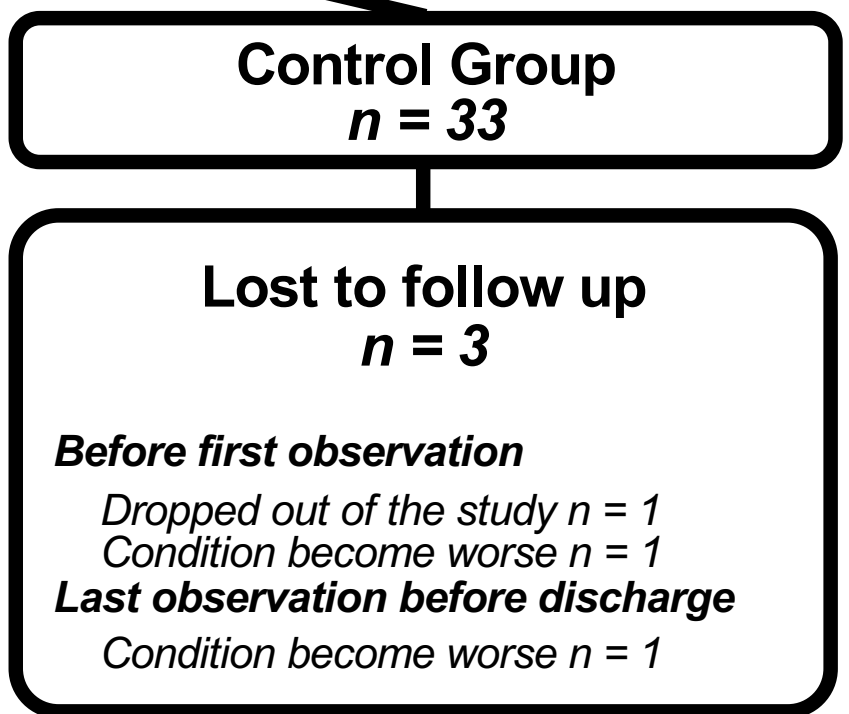

Analysed $\mathbf{n}=\mathbf{3 0}$

Excluded from analysis $n=0$

Figure 1. Flow diagram of the study

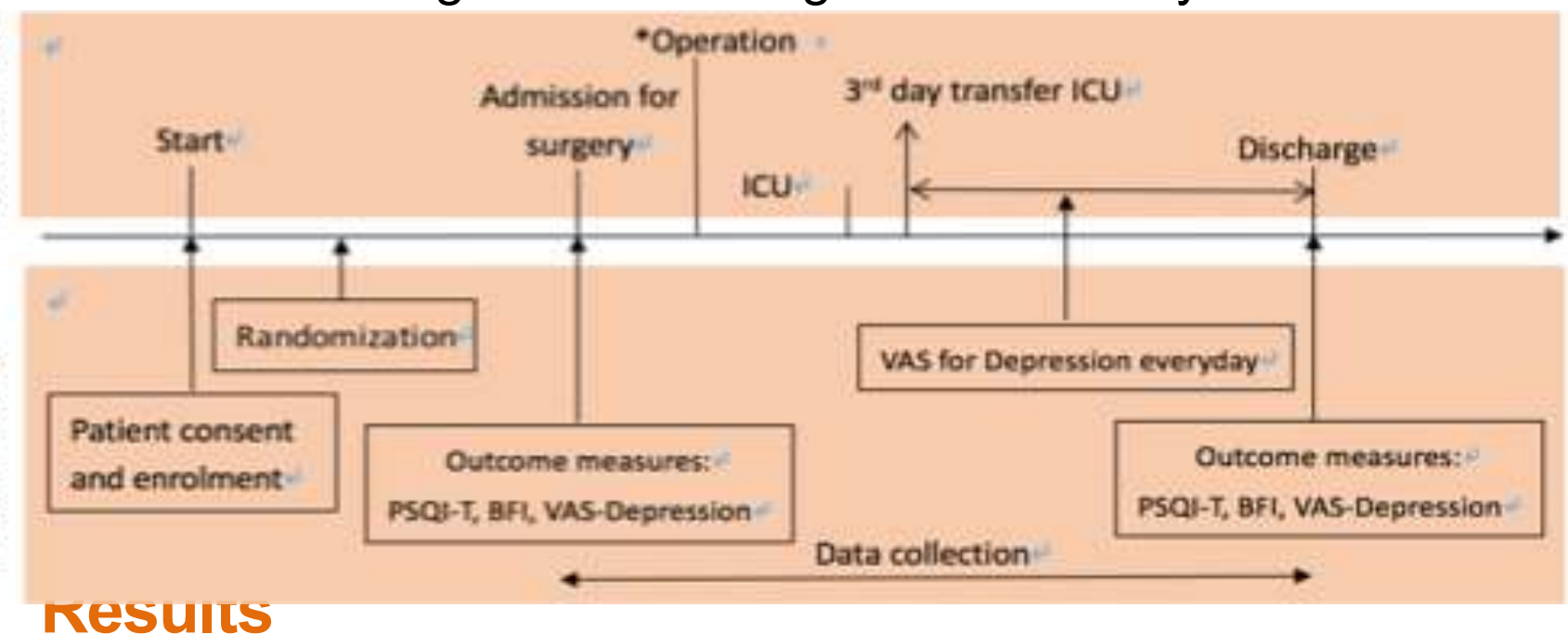

A total of 60 patients were included and analyzed(see Table 1), excluding 7 patients were dropped from the study for a few reasons. General Estimated Equation analysis revealed that the sleep quality $(\beta=-2.07 ; p<0.05)$ and fatigue $(\beta=-0.80 ; p<0.05)$ of the intervention group exhibited greater improvements than those of the control group (see Table 2). The depression level of the intervention group was significantly lower than of the control group on Days 4, Day 5, Day 7, Day 9 and Day $10(p<0.05)$ (see Figure 2).

Table 1. Characteristics of the Participants

Significant difference: " $p<05, " p<01, \cdots " p<001$

Table 2. Use generalized estimating equations analysis for comparing the sleep quality and fatigue in two group.

\begin{tabular}{|c|c|c|c|c|c|c|}
\hline \multirow{2}{*}{ parameter } & \multirow{2}{*}{$B$} & \multirow{2}{*}{$\begin{array}{l}\text { Std. } \\
\text { Error }\end{array}$} & \multicolumn{2}{|c|}{$95 \%$ Wald $\mathrm{Cl}$} & \multirow{2}{*}{$\begin{array}{c}\text { Wald } \\
\text { Chi-Square }\end{array}$} & \multirow{2}{*}{ p-value } \\
\hline & & & Lower & Upper & & \\
\hline \multicolumn{7}{|l|}{ Sleep quality } \\
\hline Intercept & 5.97 & 0.73 & 4.85 & 7.09 & 108.63 & $0.00^{* * *}$ \\
\hline $\begin{array}{l}\text { Intervention Group } \\
\text { Baseline (control Group) }\end{array}$ & 0.43 & 0.88 & -1.30 & 2.16 & 0.24 & 0.62 \\
\hline $\begin{array}{l}\text { Day } 10 \text { at ICU after surgery } \\
\text { Baseline (before Surgery) }\end{array}$ & 4.87 & 0.61 & 3.67 & 6.06 & 63.54 & $0.00 *$ \\
\hline Group * Time & -2.07 & 0.94 & -3.91 & -0.23 & 4.85 & $0.03 * *$ \\
\hline Fatigue & & & & & & \\
\hline Intercept & 0.41 & 0.19 & 0.21 & 0.80 & 4.26 & $0.04 *$ \\
\hline $\begin{array}{l}\text { Intervention Group } \\
\text { Baseline (control Group) }\end{array}$ & 0.09 & 0.33 & -0.56 & 0.74 & 0.07 & 0.79 \\
\hline $\begin{array}{l}\text { Day } 10 \text { at ICU after surgery } \\
\text { Baseline (before Surgery) }\end{array}$ & 3.03 & 0.23 & 2.57 & 3.48 & 171.99 & $0.00^{* * *}$ \\
\hline Group * Time & -0.80 & 0.38 & -1.55 & -0.06 & 4.50 & $0.03^{* *}$ \\
\hline
\end{tabular}

Figure 2. Compare the variation of depression level between the two groups

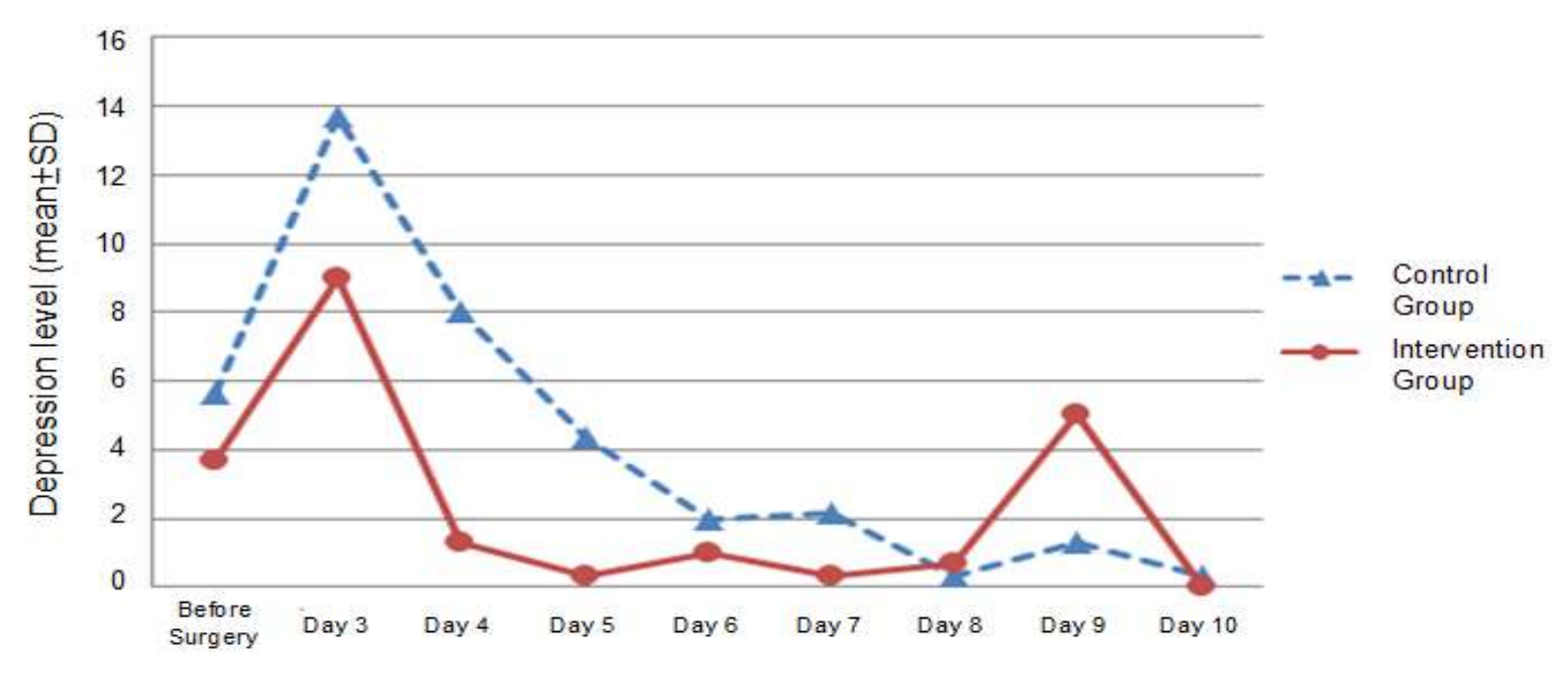

\begin{tabular}{|l|c|c|c|c|c|c|c|c|c|}
\hline & $\begin{array}{c}\text { Before } \\
\text { Surgery }\end{array}$ & Day 3 & Day 4 & Day 5 & Day 6 & Day 7 & Day 8 & Day 9 & Day 10 \\
\hline $\begin{array}{l}\text { Intervention } \\
\text { group }\end{array}$ & $4.33 \pm 15.24$ & $9.00 \pm 13.73$ & $1.33 \pm 4.34$ & $0.33 \pm 1.83$ & $1.00 \pm 4.03$ & $0.33 \pm 1.83$ & $0.67 \pm 2.54$ & $5.00 \pm 19.07$ & $0.00 \pm 0.00$ \\
\hline $\begin{array}{l}\text { Control } \\
\text { group }\end{array}$ & $6.00 \pm 12.76$ & $13.67 \pm 18.47$ & $8.00 \pm 11.57$ & $4.33 \pm 10.06$ & $2.00 \pm 9.25$ & $2.17 \pm 9.26$ & $0.33 \pm 1.83$ & $1.33 \pm 5.71$ & $0.33 \pm 1.83$ \\
\hline$p$-value & 0.56 & 0.23 & $0.00 *$ & $0.00 * \pm$ & 0.26 & $0.04 *$ & 0.24 & $0.04 *$ & $0.04 *$ \\
\hline
\end{tabular}

\section{Conclusions}

The use of muscle relaxation intervention does prove to be of greater benefit in decreasing fatigue and promote sleep quality. Hence, clinical care workers could use this results as a reference for patient care. 